\title{
Elasto-thermoelectric beam formulation for modeling thermoelectric devices
}

\author{
R. Palma ${ }^{\mathrm{a}}$, E. Moliner ${ }^{\mathrm{a}}$, J.L. Pérez-Aparicio ${ }^{\mathrm{b}}$ \\ ${ }^{a}$ Department of Mechanical Engineering and Construction, Av. Sos Baynat, s/n - Universitat Jaume I, \\ 12071 Castellón de la Plana (Spain), e-mail: rpalma@uji.es \\ ${ }^{b}$ Mecánica de Medios Continuos y Teoría de Estructuras, Universitat Politècnica de València, Spain
}

\begin{abstract}
The present paper provides a dynamic, non-linear and fully coupled Finite Element (FE) formulation based on the Timoshenko beam theory to study elasto-thermoelectric responses in thermoelectric devices. The two main motivations of this work are: i) to study mechanical responses in thermoelectric devices, which must be taken into account in the design of Peltier cells due to the fragility and relative low strength of the semiconductors, and ii) to provide a numerical tool that decreases the CPU time to allow the introduction of designs based on optimization processes and on sensitivity analyses that could require many evaluations. In order to undertake the objectives of this work, the general three-dimensional governing equations are reduced to one-dimensional ones by means of several assumptions. Then, a set of five multi-coupled partial differential equations is obtained. The resultant expressions are thermodynamically consistent and form a multi-coupled monolithic FE formulation, differently to stagger formulations that require two separated steps to reach the final result. Numerically, this set of multi-coupled equations is discretized using the FE method and implemented into FEAP [1]. For a proper validation of the code, four benchmarks are performed using one-dimensional dynamic analytical solutions developed by the authors. Finally, this formulation is compared with a three-dimensional FE formulation also developed by the authors in [2] to model a commercial Peltier cell. This comparison reveals that: i) relative errors are lower than $13 \%$ and ii) CPU times decrease significantly, more than one order of magnitude. In conclusion, the beam thermoelectric formulation is an accurate model that reduces CPU time and could be used in future design of thermoelectric devices.
\end{abstract}

Keywords: Thermoelectric, Timoshenko beam model, Finite Element Method, Thermodynamics, Peltier cells

\section{Introduction}

Thermoelectric materials, which couple thermal and electric fields, are used in many applications for cooling/heating and energy generation, see [3] for a complete review of thermoelectric applications.

The study of the thermoelectric coupling has been widely addressed from analytical, experimental and numerical points of view. However, the inclusion of the mechanical field (elasto-thermoelectric) in order to take into account thermal stresses has not been adequately studied. The main reason of this lack of research works could be that, traditionally, the thermoelectric problem has been dealt by electric engineers. The electrical engineering community usually uses one-dimensional (1D) analytical solutions that, at least, make it very difficult to couple the mechanical field. Notice that, from a mathematical point of view, the mechanical field magnitudes are represented by tensors, while electric and thermal ones by vectors. In short, the mechanical behavior of thermoelectric devices has not been well understood to date, and one of the aim of this work is to provide a simple numerical tool to study this coupling in future works.

From a numerical point of view, the authors of the present work have published several works on thermoelectric modeling, using the Finite Element (FE) method. In [4], a three-dimensional (3D) non-linear FE formulation for thermoelectric modeling was developed. The non-linearities emerge from the Joule term and the temperature dependency of the material properties. The previous formulation was applied to model a commercial Peltier device in [5]. Subsequently, in [6] the FE formulation was extended by including a temperature relaxation time based on the Cattaneo model. In [7], four relaxation times were introduced and it was numerically verified that the hysteresis-like response of thermoelectrics was due to a coupled relaxation time [8]. In the previous FE formulations, the mechanic field 
was not included. In [2] a thermodynamically consistent $3 \mathrm{D}$, non-linear and fully coupled formulation (including thermal, electric, magnetic and mechanic fields) was addressed under static and dynamic situtations. Small displacements, rotations and strains were assumed for the mechanical field. Other authors have used commercial FE codes to study thermoelectricity. For instance, Peltier devices were simulated using the commercial FE code ANSYS in [9, 10] and COMSOL in [11]. These works do not take into account the mechanical field. Conversely, in [12] a Peltier cell was analyzed using an elasto-thermoelectric FE implemented in COMSOL. This last work concludes that thermal stresses cause a mechanical bending of the thermocouples that compose the Peltier cell. Recently, the authors of [13, 14, 15] have analyzed thermal stresses in thermoelectric power generators using FE formulations. For this purpose, a stagger procedure consisting of two steps is developed. First, temperature distributions are obtained using a pure thermoelectric model implemented in ANSYS; then, these thermal distributions are introduced in a pure mechanical model. This is not a monolithic FE approach (fully coupled stiffness matrix) derived from a thermodynamically consistent formulation, as in [2]. The authors in [15] report that thermal stresses should be considered to improve the mechanical reliability of these generators.

The literature review shows that there exist still few works dealing with the elasto-thermoelectric behavior in thermoelectric devices, despite the fact that thermal stresses may significantly affect their mechanical performance and, consequently, their service life. Among others, one drawback to numerically study ther- ${ }^{103}$ mal stresses is the higher computational cost, [16]. For ${ }^{104}$ instance, the two numerical alternatives (iterative solu- 105 tions as in [15] and consistent fully coupled as in [2]) to compute thermal stresses increase the CPU time and the formulation difficulty. An alternative approach to consider thermal stresses, reducing CPU time and ensuring a consistent formulation could be derived from classic 108 mechanics of materials. For example, in [17] and [18] 109 multiphysics beam formulations to model piezoelectrics 110 and composites, respectively, are reported.

The present work presents a dynamic, non-linear 112 and fully coupled FE formulation based on the Timo- 113 shenko beam theory to study elasto-thermoelectric re- 114 sponses in thermoelectric devices. In order to obtain 115 the FE formulation, several assumptions such as small 116 strains, displacements and rotations, two-dimensional slender beam-like structures and the absence of mag- ${ }_{117}$ netic fields, convection, or radiation phenomena are in- 118 troduced. Then, this beam formulation is implemented 119

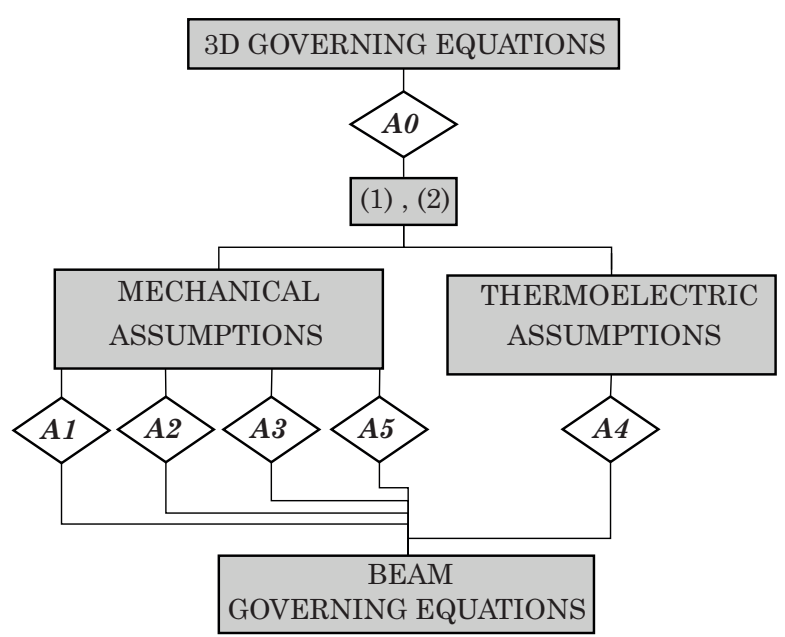

Figure 1: Starting from a 3D formulation, several assumptions are introduced to derive a thermodynamically consistent beam formulation. All theses assumptions are justified and highlighted through the work.

into FEAP [1], a FE analysis program developed by the University of Berkeley at California (USA). For the validation of the implementation, four benchmarks are performed using 1D dynamic analytical solutions of a single thermoelement. Despite the fact that bending is not present in thermoelectric thermoelements, the bending could be relevant in Peltier devices due to the framelike behavior of these devices. For this purpose, the present formulation is compared with the $3 \mathrm{D}$ one developed by the authors in [2] to model a commercial Peltier device. This comparison reveals that: i) relative errors are lower than $13 \%$ and ii) CPU times decrease significantly, more than one order of magnitude. In conclusion, the beam thermoelectric formulation is an accurate model and could be used in future optimizations and sensitivity analyses that require many evaluations.

\section{Governing equations}

The aim of this section is to establish the governing equations that are used for the FE formulation. Starting from a 3D set of equations, several assumptions or simplifications are considered to derive a thermodynamically consistent beam formulation. The assumptions through the document are highlighted in text-box and are denoted by $\boldsymbol{A 1}, \boldsymbol{A 2}$, etc. Furthermore, for the sake of clarity the complete set of assumptions is represented in the flowchart shown in Figure 1.

\subsection{Outline of $3 D$ governing equations}

This section outlines the 3D elasto-thermoelectric governing equations, composed of three balance equa- 
tions, three constitutive (also called transport) equations $\quad 154$ and six boundary conditions. In addition, one compati- 155 bility equation is considered for the mechanical field.

\section{Assumption A0: Small strains and displacements.}

In most applications, $\boldsymbol{A O}$ is a first and good approximation due to the high stiffness of typical thermoelectric materials. Considering this assumption, the non-local strong forms of the balance equations are expressed as follow:

$$
\begin{aligned}
0 & =\int_{\Omega}\left(\nabla \cdot \boldsymbol{\sigma}+\boldsymbol{b}-\rho_{m} \ddot{\boldsymbol{u}}\right) \mathrm{d} \Omega \\
0 & =\int_{\Omega}\left(-\nabla \cdot \boldsymbol{q}-\boldsymbol{j} \cdot \nabla V-T_{0} \boldsymbol{\beta}: \dot{\boldsymbol{\epsilon}}-\rho_{m} c \dot{T}\right) \mathrm{d} \Omega, \\
0 & =\int_{\Omega} \nabla \cdot \boldsymbol{j} \mathrm{d} \Omega
\end{aligned}
$$

where $\Omega$ denotes 3D domain, $\rho_{m}$ mass density, $\boldsymbol{u}$ displacement vector, $\boldsymbol{\sigma}$ stress tensor, $\boldsymbol{b}$ vector of body forces, $c$ heat capacity, $T$ temperature, $\boldsymbol{q}$ heat flux, $\boldsymbol{j}$ electric flux, $V$ voltage, $T_{0}$ reference temperature, $\boldsymbol{\beta}$ thermal expansion tensor (in Lamé form) and $\epsilon$ denotes strain tensor. In addition, ( $\left.{ }^{\circ}\right)$ and (') represent first and second time derivatives. The first equation is the linear momentum balance required to ensure the mechanical equilibrium. Furthermore, the angular momentum balance requires that $\sigma$ is symmetric. The second equation is the energy balance and takes into account the energy of the three fields: the first term on the right side represents the thermal energy that flows across the boundary; the second and third terms are sources due to electric field (Joule heating) and mechanical field (Biot or twoway effect, see [2], [19], [20] and [21]), respectively. ${ }^{178}$ Finally, the third equation states the balance of electric 179 charge and is obtained by combining the Ampère and 180 Gauss laws of electromagnetism. Free electric charges ${ }^{181}$ are not considered in the present work and, therefore, 182 the left term is zero.

The constitutive equations are a set of three coupled ${ }_{183}$ equations given by:

$$
\begin{aligned}
\boldsymbol{\sigma} & =\mathbf{C}: \boldsymbol{\epsilon}-\boldsymbol{\beta}\left(T-T_{0}\right) \\
\boldsymbol{q} & =-\kappa(T) \nabla T+\alpha(T) T \boldsymbol{j}, \\
\boldsymbol{j} & =-\gamma(T) \nabla V-\alpha(T) \gamma(T) \nabla T,
\end{aligned}
$$

where $\mathbf{C}$ denotes elastic tensor, $\kappa$ thermal conductivity, 189 $\alpha$ Seebeck coefficient and $\gamma$ denotes electric conductiv- 190 ity. The first equation describes the thermoelastic cou- 191 pling; electric and mechanic fields are not coupled since 192 polarization effects (such as piezoelectric interactions) are not usually present in thermoelectric devices. The second and third equations couple thermal and electric fields by two separate effects, Seebeck and Peltier, both measured by the Seebeck coefficient.

Although there is not an explicit relationship between electric and mechanic fields, the problem is fully coupled since both depend on temperature.

In most practical situations, the materials are isotropic and homogeneous. Furthermore, $\kappa, \gamma, \alpha$ typically depend on temperature, resulting material nonlinearities. According to [5], the temperature dependency of material properties can be fitted using secondorder polynomials to obtain:

$$
\begin{aligned}
& \kappa(T)=\kappa_{0}+\kappa_{1} T+\kappa_{2} T^{2}, \\
& \gamma(T)=\gamma_{0}+\gamma_{1} T+\gamma_{2} T^{2}, \\
& \alpha(T)=\alpha_{0}+\alpha_{1} T+\alpha_{2} T^{2},
\end{aligned}
$$

where $\kappa_{i}, \gamma_{i}, \alpha_{i}$ are coefficients reported in [5].

As commented, the mechanical field requires a compatibility equation that relates displacement vector and the strain tensor and is given by:

$$
\boldsymbol{\epsilon}=\nabla^{s y} \boldsymbol{u},
$$

where $(\cdot)^{s y}$ denotes the symmetric part of the displacement gradient. Notice that the skew-symmetric part represents the rigid body rotations and, therefore, do not contribute to the strain measure.

Finally, a set of six boundary conditions (3 Neumanntype and 3 Dirichlet-type) must be considered:

$$
\begin{array}{ll}
\boldsymbol{\sigma} \cdot \mathbf{n}=\boldsymbol{t} & ; \quad \boldsymbol{u}=\overline{\boldsymbol{u}} \\
\boldsymbol{q} \cdot \mathbf{n}=q_{c} \quad ; \quad T=\bar{T} \\
\boldsymbol{j} \cdot \mathbf{n}=j_{c} \quad ; \quad V=\bar{V} \quad,
\end{array}
$$

where $\mathbf{n}$ denotes outward normal to the boundary, $\boldsymbol{t}$ traction vector, $q_{c}$ and $j_{c}$ denote heat and electric fluxes at boundary, respectively, and $\overline{\boldsymbol{u}}, \bar{T}, \bar{V}$ denote the prescribed displacement, temperature and voltage, respectively.

\subsection{Elasto-thermoelectric beam equations}

In this section, the $3 \mathrm{D}$ governing equations are simplified to the Timoshenko beam model. The choice of this model intends to find a compromise between generality and simplicity of implementation. The former is achieved since this beam model is more general than that of Bernoulli: it takes into account shear stresses and strains by considering an extra rotation, a degree of freedom coupled with displacements. The simplicity arises from the fully coupled formulation of the present work 


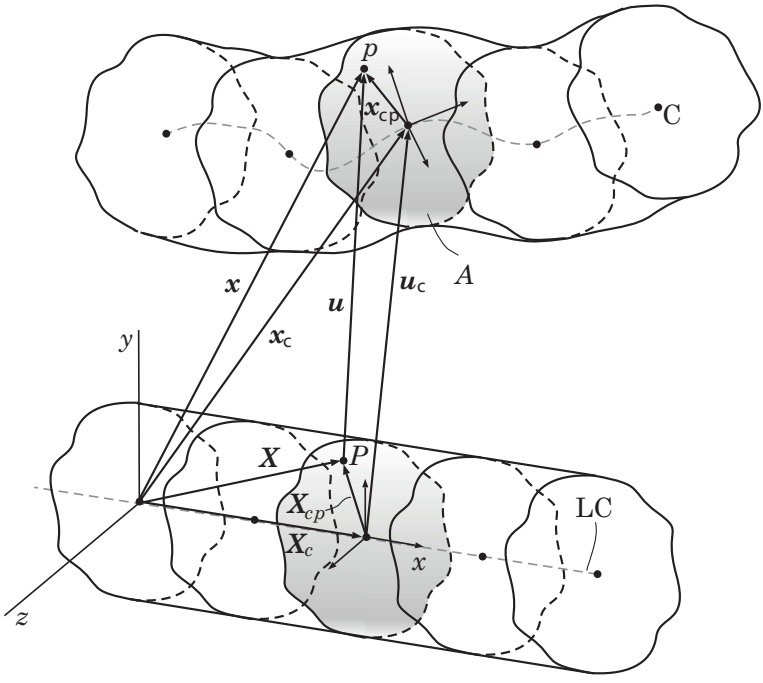

Figure 2: Beam before (bottom) and after (top) deformation. The beam is represented as a long body composed of a succession of $2 \mathrm{D}$ cross sections, of area $A$, attached at their centroid (C) to a longitudinal axis called line of centroids (LC).

in which the inclusion of the extra rotation is comparable in difficulty to that of temperature and voltage.

The Timoshenko beam is a well established model ${ }^{233}$ from a century ago, [22], but it is here briefly reviewed ${ }^{234}$ to introduce the coupled formulations: in particular, the ${ }^{235}$ understanding of the coupling between rotational and translational degrees of freedom (dof) is a good introduction for the understanding of the coupling between mechanical, on one side, and voltage and temperature dof's the other.

\subsubsection{Kinematics and thermoelectric distributions}

A beam is a long body composed of a succession of 2D planar surfaces called cross section, of area $A$. The ${ }^{241}$ surfaces are attached in their centroid to a longitudinal axis perpendicular to each cross section, called line of ${ }^{242}$ centroids (LC).

The beam motion is constrained by the kinematic 244 beam hypothesis (assumption 1, denoted by A1):

Assumption A1: A cross section that is plane before deformation remains plane after.

According to $\mathbf{A 1}$, the cross section moves as a rigid body: neither changes its shape nor deviates from flatness. Therefore, the motion of beams can be described as the deformation of the LC plus the rigid rotation of the cross section.

Figure 2 shows a beam before and after deformation with LC along the $x$ axis and with cross section of area
$A$ and contour $\Gamma$. The position of the cross section centroid $\mathrm{C}$ and of one point $P$ inside are described by $\boldsymbol{X}_{c}(x)$, $\boldsymbol{X}_{c p}(y, z)$ before and by $\boldsymbol{x}_{c}(x), \boldsymbol{x}_{c p}(y, z)$ after deformation, respectively. The origin of the first is the coordinate center, of the second the centroid itself.

From vector calculus:

$$
\begin{aligned}
\boldsymbol{X} & =\boldsymbol{X}_{c}(x)+\boldsymbol{X}_{c p}(y, z), \\
\boldsymbol{x} & =\boldsymbol{x}_{c}(x)+\boldsymbol{x}_{c p}(y, z) .
\end{aligned}
$$

According to the Chasles' theorem, the rigid rotation of $\boldsymbol{X}_{c p}$ is expressed by:

$$
\boldsymbol{x}_{c p}=\boldsymbol{R} \cdot \boldsymbol{X}_{c p},
$$

where $\boldsymbol{R}$ is the rotational operator given by $\boldsymbol{R}=\boldsymbol{I}+$ $\boldsymbol{\Theta}+$ hot; hot is the abbreviation of high order terms, $\boldsymbol{\Theta}$ is the skew-symmetric spin tensor that can be expressed as $\boldsymbol{\Theta}=\boldsymbol{\epsilon} \cdot \boldsymbol{\theta}$, where $\boldsymbol{\epsilon}$ is the Levi-Civita symbol and $\boldsymbol{\theta}$ is an axial vector of rotations (also called spin vector).

\section{Assumption A2: Small rotations are considered: $\boldsymbol{R} \approx \boldsymbol{I}+\boldsymbol{\Theta}$}

In the community of Continuum Mechanics, the displacement vector is given by $\boldsymbol{u}=\boldsymbol{x}-\boldsymbol{X}$. Therefore, the beam displacement vector is obtained by using (6), (7) and applying $\boldsymbol{A} \boldsymbol{2}$ to read:

$$
\boldsymbol{u}=\boldsymbol{u}_{c}(x)+\boldsymbol{\theta}(x) \times \boldsymbol{X}_{c p}(y, z)
$$

where the spin vector depends on the position of the cross section, $x$. As commented, in (8) the displacement is composed of an LC deformation (first term on the right side) and a rigid rotation of the cross section (second term).

\section{Assumption A3: 2D beams are considered.}

In most thermoelectric applications, the geometry of the cross section is constant and symmetric respect to both axes $y, z$ and the loads are only applied along $x$ and/or $y$ directions. According to $\boldsymbol{A 3}$ the displacements in (8) are reduced to:

$$
\left\{\begin{array}{l}
u=u_{c}(x)-\theta y, \\
v=v_{c}(x) .
\end{array}\right.
$$

Regarding the kinematic-like description of temperature and voltage distributions, Taylor series expansions evaluated at the centroid $C$ are used [17]:

$$
\begin{aligned}
& T(x, y, z)=T_{c}(x)+y \partial_{y} T+z \partial_{z} T+\text { hot } \\
& V(x, y, z)=V_{c}(x)+y \partial_{y} V+z \partial_{z} V+h o t
\end{aligned}
$$


where $\partial_{y}, \partial_{z}$ denote partial derivatives with respect to $y, z$, respectively. Notice that the thermoelectric beam simplification is similar to the mechanic one given by (8). Temperature and voltage distributions are composed of centroidal terms $T_{c}, V_{c}$ and across the section ones, given by second and third terms on the right side in (10). The terms across the section are not considered in the present work due to $\boldsymbol{A 4}$.

\section{Assumption A4: Voltage and temperature distri-} butions through cross section are constant.

The assumption $\boldsymbol{A 4}$ implies that $T(x, y, z)={ }_{283}$ $T_{c}(x), V(x, y, z)=V_{c}(x)$ and it is a good approximation for Peltier devices in the absence of magnetic field, convection and radiation phenomena, as can be extracted from the 3D FE model reported in [2].

\subsubsection{Strain-like measures}

The strain measures are obtained by operating the 3D compatibility equation (4) and using the displacement vector (9). Then, the non-zero entries in $\epsilon$ are given by:

$$
\left\{\begin{array}{l}
\varepsilon_{x x}=\partial_{x} u_{c}-\partial_{x} \theta y \\
\varepsilon_{x y}=\frac{1}{2}\left(\partial_{x} v_{c}-\theta\right),
\end{array}\right.
$$

where $\partial_{x}$ denote partial derivative with respect to $x$. As ${ }^{289}$ is typical in beam theory, three new strain measures $\varepsilon_{x},{ }^{290}$ $\varepsilon_{y}, \varepsilon_{\theta}$ are defined as:

$$
\left\{\begin{array}{l}
\varepsilon_{x}=\partial_{x} u_{c}, \\
\varepsilon_{y}=\partial_{x} v_{c}-\theta, \\
\varepsilon_{\theta}=-\partial_{x} \theta
\end{array}\right.
$$

Using these new definitions, (11) becomes:

$$
\left\{\begin{array}{l}
\varepsilon_{x x}=\varepsilon_{x}+\varepsilon_{\theta} y \\
\varepsilon_{x y}=\frac{1}{2} \varepsilon_{y} .
\end{array}\right.
$$

Regarding temperature and voltage strain-like ${ }_{20} 250$ sures, the general 3D gradients are reduced to:

$$
\begin{aligned}
& \nabla T \approx \partial_{x} T_{c}, \\
& \nabla V \approx \partial_{x} V_{c} .
\end{aligned}
$$

\subsubsection{Equilibrium equations}

From an equilibrium point of view, the difference between 3D and beam formulations arises from the concept of stress resultant. That is, the tractions $t$ in any point of the cross section are expressed by the force 301 $\boldsymbol{F}$ and the momentum $\boldsymbol{M}$ resultants at the center of the 302 cross section, see Figure 3 (left). Mathematically, con- 303 sidering that $\mathbf{n} \equiv(1,0,0)$ is the outward normal to the ${ }_{304}$
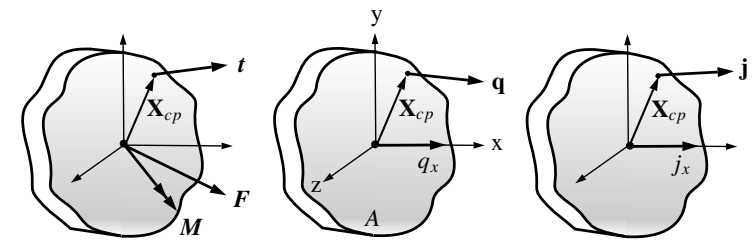

Figure 3: From 3D to 1D. Beam models use resultants integrating through cross section area: force and moment (left), amount of heat along $x$ (middle) and electric intensity along $x$ (right).

cross section, the force and momentum resultants are given by:

$$
\begin{gathered}
\boldsymbol{F}=\int_{A} \boldsymbol{t} \mathrm{d} A=\int_{A} \boldsymbol{\sigma} \cdot \mathbf{n} \mathrm{d} A=N \hat{\mathbf{x}}+Q \hat{\mathbf{y}}+Q_{z} \hat{\mathbf{z}}, \\
\boldsymbol{M}=\int_{A} \boldsymbol{x}_{c p} \times(\boldsymbol{\sigma} \cdot \mathbf{n}) \mathrm{d} A=M_{x} \hat{\mathbf{x}}+M_{y} \hat{\mathbf{y}}+M \hat{\mathbf{z}},
\end{gathered}
$$

where $N, Q, Q_{z}$ are axial and shear forces and $M_{x}, M_{y}$, $M$ are the moment respect to the unit vectors $\hat{\mathbf{x}}, \hat{\mathbf{y}}, \hat{\mathbf{z}}$ axis, respectively. Notice that $Q_{z}, M_{x}, M_{y}$ are neglected due to $\boldsymbol{A 3}$ and since the cross section is symmetric respect to both axes $y, z$ in most thermoelectric applications.

In order to ensure the mechanical equilibrium, linear and angular momenta must be stated by particularizing (1) to the beam model, see [23]. Then, a set of three partial differential equations is obtained:

$$
0=\int_{x}\left[\frac{\partial}{\partial x}\left\{\begin{array}{c}
N \\
Q \\
M
\end{array}\right\}+\left\{\begin{array}{c}
f_{u}-\rho_{m} A \ddot{u}_{c} \\
f_{v}-\rho_{m} A \ddot{v}_{c} \\
Q+m-\rho_{m} I \ddot{\theta}
\end{array}\right\}\right] \mathrm{d} x,
$$

where $f_{u}, f_{v}, m$ are distributed applied forces and moment and $I$ denotes second moment of area.

Thermal and electric fluxes must be reduced to 1D following a similar procedure to that described to obtain force and momentum resultants. Denoting by $Q$ the amount of heat transferred per unit time and by $\mathcal{I}$ the electric intensity that flows along $x, q_{x}, j_{x}$ are expressed as:

$$
Q=\int_{A} \boldsymbol{q} \cdot \mathbf{n} \mathrm{d} A=A q_{x} \quad, \quad \mathcal{I}=\int_{A} \boldsymbol{j} \cdot \mathbf{n} \mathrm{d} A=A j_{x},
$$

where to solve the integral it is considered that cross sections are constant, Figure 3 middle and right. Introducing this $1 \mathrm{D}$ approximation in the second, third equations of (1): 


$$
0=\int_{x}\left[\frac{\partial}{\partial x}\left\{\begin{array}{c}
-Q \\
\mathcal{I}
\end{array}\right\}-\left\{\begin{array}{c}
\mathcal{J}+\mathcal{T}+A \rho_{m} c \dot{T}_{c} \\
0
\end{array}\right\}\right] \mathrm{d} x
$$

where, for the sake of clarity, the terms due to Joule $\mathcal{J}$ and to two-way $\mathcal{T}$ have been denoted by:

$$
\mathcal{J}=\mathcal{I} \partial_{x} V_{c} \quad, \quad \mathcal{T}=T_{0} A E \alpha_{T} \dot{\varepsilon}_{x}
$$

respectively. Notice that $\boldsymbol{F}, \boldsymbol{M}$ are vectors since the stress tensor is a second order tensor and $Q, \mathcal{I}$ are scalars due to the fact that heat and electric fluxes are vectors.

\subsubsection{Constitutive equations}

Assumption A5: The Poisson effect is neglected.

Due to the kinematic approximation of the Timoshenko beam model, the non-zero components of $\epsilon$ are given in (11). This simplification results in non-zero spurious stresses $\sigma_{y y}, \sigma_{z z}$ usingconstitutive equations. In order to fix this inconsistency, both stress components are forced to zero. Using the Hooke's constitutive equations in Lamé form, see [23], the result is $\sigma_{x x}=E \epsilon_{x x}$. Comparing the values of all stress entries, $(2 G+\lambda)=E$; this expression is equivalent to neglect the Poisson coefficient in the definition of $G$.

Using (13), the first equation in (2) and taking into account $\boldsymbol{A 5}$, the 1D thermoelastic constitutive equation ${ }^{34}$ becomes:

$$
\left\{\begin{array}{l}
\sigma_{x x}=E\left(\varepsilon_{x}+\varepsilon_{\theta} y-\alpha_{T} \Delta T\right) \\
\sigma_{x y}=G \varepsilon_{y}
\end{array}\right.
$$

where $\Delta T=T_{c}-T_{0}, \alpha_{T}$ is the thermal expansion coef- 352 ficient. Finally, using (15) and, again, considering con- 353 stant cross sections, the 1D thermoelastic constitutive 354 equations are given by:

$$
\left\{\begin{array}{c}
N \\
Q \\
M
\end{array}\right\}=\int_{A}\left\{\begin{array}{c}
T_{x x} \\
T_{x y} \\
-T_{x x} y
\end{array}\right\} \mathrm{d} A=\left\{\begin{array}{c}
\left.A E\left[\varepsilon_{x}-\alpha_{T} \Delta T\right)\right] \\
k_{s} A G \varepsilon_{y} \\
-E I \varepsilon_{\theta}
\end{array}\right\},
$$

where the first moment of area $\int_{A} y \mathrm{~d} A=0$ since $y, z$ cross the centre of gravity. The Timoshenko shear factor, which is typically $5 / 6$ for rectangular cross-sections [24], is denoted by $k_{s}$.

Similarly, the second and third 3D thermoelectric 358 constitutive equations (2) are simplified to 1D relation- 359 ships by considering (17), to give:

$$
\begin{aligned}
Q & =-A \kappa\left(T_{c}\right) \partial_{x} T_{c}+\alpha\left(T_{c}\right) T_{c} \mathcal{I}, \\
\mathcal{I} & =-A \gamma\left(T_{c}\right) \partial_{x} V_{c}-A \alpha\left(T_{c}\right) \gamma\left(T_{c}\right) \partial_{x} T_{c} .
\end{aligned}
$$

\section{Finite Element formulation}

The current section presents the discretisations based on the FE method to solve the set of five fully coupled partial differential equations described in Section 2. As commented and since the problem is multi-coupled, there are five dof's: $u_{c}, v_{c}, \theta, T_{c}$ and $V_{c}$.

\subsection{Weak forms}

The balance equations given in (16) and (18) are expressed in weak form following the standard FE procedure, namely, multiplying by test functions and applying the divergence theorem to obtain:

$-\int_{x}\left[\frac{\partial}{\partial x}\left\{\begin{array}{l}\delta u_{c} N \\ \delta v_{c} Q \\ \delta \theta M \\ \delta T_{c} Q \\ \delta V_{c} \mathcal{I}\end{array}\right\}-\left\{\begin{array}{l}\delta u_{c}\left(n-\rho_{m} A \ddot{u}_{c}\right) \\ \delta v_{c}\left(q-\rho_{m} A \ddot{v}_{c}\right) \\ \delta \theta\left(Q+m-\rho_{m} I \ddot{\theta}\right) \\ \delta T_{c}\left(A \rho_{m} c \dot{T}_{c}+\mathcal{J}+\mathcal{T}\right) \\ 0\end{array}\right\}\right] \mathrm{d} x=0$.

\subsection{Residual forms}

Since the problem is non-linear due to the presence of the Joule term and the temperature dependency of the material properties given in (3), the FE formulation is expressed in residual forms to use the Newton-Raphson algorithm.

Firstly, the continuum weak form calculated in (23) is discretized by using the same standard 1D shape functions of Lagrange type for all the degrees of freedom, since the problem holds continuity $C^{0}$ :

$$
\left\{\begin{array}{c}
\delta u_{c} \\
\delta v_{c} \\
\delta \theta \\
\delta T_{c} \\
\delta V_{c}
\end{array}\right\} \approx \mathcal{N}_{a}\left\{\begin{array}{c}
\delta \mathrm{a}_{a}^{u} \\
\delta \mathrm{a}_{a}^{v} \\
\delta \mathrm{a}_{a}^{\theta} \\
\delta \mathrm{a}_{a}^{T} \\
\delta \mathrm{a}_{a}^{V}
\end{array}\right\} ; \partial_{x}\left\{\begin{array}{c}
\delta u_{c} \\
\delta v_{c} \\
\delta \theta \\
\delta T_{c} \\
\delta V_{c}
\end{array}\right\} \approx \mathcal{B}_{a}\left\{\begin{array}{c}
\delta \mathrm{a}_{a}^{u} \\
\delta \mathrm{a}_{a}^{v} \\
\delta \mathrm{a}_{a}^{\theta} \\
\delta \mathrm{a}_{a}^{T} \\
\delta \mathrm{a}_{a}^{V}
\end{array}\right\},
$$

where $\delta \mathrm{a}_{a}^{i}$ denotes the virtual nodal value of the dofs $i=u_{c}, v_{c}, \theta, T_{c}, V_{c}$ at node $a$, and $\mathcal{B}_{a}=\partial_{x} \mathcal{N}_{a}$.

Secondly, the residuals at node $a$ are obtained by introducing (24) in (23): 
$\left\{\begin{array}{l}\mathcal{R}_{a}^{u} \\ \mathcal{R}_{a}^{v} \\ \mathcal{R}_{a}^{\theta} \\ \mathcal{R}_{a}^{T} \\ \mathcal{R}_{a}^{V}\end{array}\right\}=-\int_{x^{e}}\left[\mathcal{B}_{a}\left\{\begin{array}{c}N \\ Q \\ M \\ \mathcal{Q} \\ \mathcal{I}\end{array}\right\}-\mathcal{N}_{a}\left\{\begin{array}{l}n-\rho_{m} A \ddot{u}_{c} \\ q-\rho_{m} A \ddot{v}_{c} \\ Q+m-\rho_{m} I \ddot{\theta} \\ A \rho_{m} c \dot{T}_{c}+\mathcal{J}+\mathcal{T} \\ 0\end{array}\right\}\right] \begin{array}{cl}\frac{\partial N}{\partial \mathrm{a}_{b}^{u}}=A E \mathcal{B}_{b} & , \frac{\partial N}{\partial \mathrm{a}_{b}^{T}}=-A E \alpha_{T} \mathcal{N}_{b}, \\ \mathrm{~d} x^{e} \cdot \frac{\partial Q}{\partial \mathrm{a}_{b}^{v}}=k_{s} A G \mathcal{B}_{b} & , \frac{\partial Q}{\partial \mathrm{a}_{b}^{\theta}}=-k_{s} A G \mathcal{N}_{b},\end{array}$

\subsection{Tangent matrices}

The tangent matrices are obtained by deriving the

$$
\mathcal{K}_{a b}^{i j}=-\frac{\partial \mathcal{R}_{a}^{i}}{\partial \mathrm{a}_{b}^{j}} \quad, \quad C_{a b}^{i j}=-\frac{\partial \mathcal{R}_{a}^{i}}{\partial \dot{\mathrm{a}}_{b}^{j}} \quad, \quad \mathcal{M}_{a b}^{i j}=-\frac{\partial \mathcal{R}_{a}^{i}}{\partial \ddot{\mathrm{a}}_{b}^{j}},
$$

where, again, $i, j$ denote the dof; $a, b$ the nodes and $\mathcal{K}$, $C, \mathcal{M}$ the stiffness, capacity and mass matrices, respectively.

The stiffness matrices are calculated by:

$$
\begin{aligned}
& \frac{\partial M}{\partial \mathrm{a}_{b}^{\theta}}=-E I \mathcal{B}_{b}, \\
& \frac{\partial \mathcal{I}}{\partial \mathrm{a}_{b}^{V}}=-A \gamma\left(T_{c}\right) \mathcal{B}_{b} \quad, \quad \frac{\partial Q}{\partial \mathrm{a}_{b}^{V}}=\alpha\left(T_{c}\right) T_{c} \frac{\partial \mathcal{I}}{\partial \mathrm{a}_{b}^{V}},
\end{aligned}
$$$$
\frac{\partial \mathcal{J}}{\partial \mathrm{a}_{b}^{V}}=\frac{\partial \mathcal{I}}{\partial \mathrm{a}_{b}^{V}} \mathcal{B}_{b} \mathrm{a}_{b}^{V}+\mathcal{I} \mathcal{B}_{b},
$$$$
\frac{\partial \mathcal{J}}{\partial \mathrm{a}_{b}^{T}}=\frac{\partial \mathcal{I}}{\partial \mathrm{a}_{b}^{T}} \mathcal{B}_{b} \mathrm{a}_{b}^{V}
$$$$
\frac{\partial \mathcal{I}}{\partial \mathrm{a}_{b}^{T}}=-A \frac{\partial \gamma}{\partial T} \mathcal{N}_{b} \mathcal{B}_{b} \mathrm{a}_{b}^{V}-A \frac{\partial \alpha}{\partial T} \mathcal{N}_{b} \gamma\left(T_{c}\right) \mathcal{B}_{b} \mathrm{a}_{b}^{T}
$$

$$
\left\{\begin{array}{c}
\mathcal{K}_{a b}^{u j} \\
\mathcal{K}_{a b}^{v j} \\
\mathcal{K}_{a b}^{\theta j} \\
\mathcal{K}_{a b}^{T j} \\
\mathcal{K}_{a b}^{V j}
\end{array}\right\}=\int_{x^{e}} \mathcal{B}_{a}\left\{\begin{array}{c}
\frac{\partial N}{\partial \mathrm{a}_{b}^{j}} \\
\frac{\partial Q}{\partial \mathrm{a}_{b}^{j}} \\
\frac{\partial M}{\partial \mathrm{a}_{b}^{j}} \\
\frac{\partial Q}{\partial \mathrm{a}_{b}^{j}} \\
\frac{\partial Q}{\partial \mathrm{a}_{b}^{j}} \\
\frac{\partial \mathcal{I}}{\partial \mathrm{a}_{b}^{j}}
\end{array}\right\} \mathrm{d} x^{e}-\int_{x^{e}} \mathcal{N}_{a}\left\{\begin{array}{c}
0 \\
0 \\
\frac{\partial Q}{\partial \mathrm{a}_{b}^{j}} \\
\frac{\partial \mathcal{J}}{\partial \mathrm{a}_{b}^{j}} \\
0
\end{array}\right\} \mathrm{d} x^{e},
$$

where the non-zero derivatives are:

$$
\begin{aligned}
& -A \alpha\left(T_{c}\right) \frac{\partial \gamma}{\partial T} \mathcal{N}_{b} \mathcal{B}_{b} \mathrm{a}_{b}^{T}-A \alpha\left(T_{c}\right) \gamma\left(T_{c}\right) \mathcal{B}_{b}, \\
\frac{\partial Q}{\partial \mathrm{a}_{b}^{T}}=-A & \frac{\partial \kappa}{\partial T} \mathcal{N}_{b} \mathcal{B}_{b} \mathrm{a}_{b}^{T}-A \kappa\left(T_{c}\right) \mathcal{B}_{b} \\
& +\frac{\partial \alpha}{\partial T} \mathcal{N}_{b} T_{c} \mathcal{I}+\alpha\left(T_{c}\right) \mathcal{N}_{b} \mathcal{I}+\alpha\left(T_{c}\right) T_{c} \frac{\partial \mathcal{I}}{\partial \mathrm{a}_{b}^{T}} .
\end{aligned}
$$

The capacity matrices are due to the Biot term and to the heat transient. For this reason, they are closely related to the thermal residual and are given by:

$$
\left\{\begin{array}{l}
C_{a b}^{T u} \\
C_{a b}^{T T}
\end{array}\right\}=-\int_{x^{e}} \mathcal{N}_{a}\left\{\begin{array}{l}
T_{0} A E \alpha_{T} \mathcal{B}_{b} \\
\rho_{m} c A \mathcal{N}_{b}
\end{array}\right\} \mathrm{d} x^{e} .
$$

Finally, the mass matrices emerge from the mechanical inertia and they are obtained from the mechanical residuals, three first equations in (25):

$$
\left\{\begin{array}{c}
\mathcal{M}_{a b}^{u u} \\
\mathcal{M}_{a b}^{v v} \\
\mathcal{M}_{a b}^{\theta \theta}
\end{array}\right\}=\int_{x^{e}} \mathcal{N}_{a} \rho_{m}\left\{\begin{array}{c}
A \\
A \\
I
\end{array}\right\} \mathcal{N}_{b} \mathrm{~d} x^{e}
$$

This FE formulation is implemented into the research code FEAP [1]. This code provides several dummy routines (user elements) that can be used for the implementation of new developed modular elements written in Fortran. In order to solve the non-linear problem, the 
Newton-Raphson algorithm is used and the Newmark- $\beta$ for time integration of the dynamic. Notice that since the problem is multi-coupled, several orders of the time derivatives are present. This drawback is solved by using a special command implemented in FEAP. Finally, the shear locking, which is typically present in the Timoshenko beam models $[25,26]$, is solved due to the facilities of the FEAP code.

\section{Validations}

This section presents four validations (called cases I, II, III and IV) to ensure a proper implementation of the numerical formulation developed in Sec. 3. For this purpose, the numerical results are compared with dynamic 1D analytical solutions developed by the authors; most of the analytical solutions are reported in [6].

\begin{tabular}{|c|l|l|}
\hline Property & Value & Units \\
\hline \hline$\kappa_{0}, \kappa_{1}, \kappa_{2}$ & $1.663,-3.58 \mathrm{e}-3,-3.19 \mathrm{e}-5$ & {$\left[\mathrm{~W} / \mathrm{m}^{\circ} \mathrm{C}\right]$} \\
\hline$\gamma_{0}, \gamma_{1}, \gamma_{2}$ & $1.09 \mathrm{e} 5,-5.59 \mathrm{e} 2,2.49$ & {$[\mathrm{~A} / \mathrm{Vm}]$} \\
\hline$\alpha_{0}, \alpha_{1}, \alpha_{2}$ & $1.98 \mathrm{e}-4,3.53 \mathrm{e}-7,7.52 \mathrm{e}-10$ & {$\left[\mathrm{~V} /{ }^{\circ} \mathrm{C}\right]$} \\
\hline$E, G$ & $4.70 \mathrm{e} 10,1.68 \mathrm{e} 10$ & {$\left[\mathrm{~N} / \mathrm{m}^{2}\right]$} \\
\hline$\rho_{m}$ & $7.53 \mathrm{e} 3$ & {$\left[\mathrm{Kg} / \mathrm{m}^{3}\right]$} \\
\hline$c$ & 544 & {$[\mathrm{~J} / \mathrm{KgK}]$} \\
\hline$\alpha_{T}$ & $5.37 \mathrm{e}-6$ & {$\left[{ }^{\circ} \mathrm{C}^{-1}\right]$} \\
\hline
\end{tabular}

Table 1: p-type bismuth telluride thermoelement properties. For the n-type thermoelement properties are equal, except for $\alpha_{0}, \alpha_{1}, \alpha_{2}$ with negative sign.

For all the validations, a single p-type bismuth telluride pulsed thermoelement as that described in [16] of dimensions $5.8 \times 1.4 \times 1.4[\mathrm{~mm}]$ and properties given in Table 1 is modeled. Figure 4 shows the p-type 3D ther- ${ }_{429}$ moelement, its $1 \mathrm{D}$ beam representation and the bound- 430 ary conditions used for the validations. From a mechan- 431 ical point of view, the boundary conditions correspond ${ }_{432}$ to a cantilever beam. Thermally, the temperatures are ${ }_{433}$ fixed at both ends of the thermoelement: at cold and hot ${ }_{434}$ sides $T_{c o}=30\left[{ }^{\circ} \mathrm{C}\right]$ and $T_{h o}=80\left[{ }^{\circ} \mathrm{C}\right]$, respectively. Fi- ${ }_{435}$ nally, for the electric field the voltage is set to zero at the ${ }_{436}$ cold side. For cases II, III and IV, an electric intensity ${ }_{437}$ of $\mathcal{I}=2$ [A] is applied.

Table 2 summarizes all validations. For case I, the ${ }_{439}$ prescribed intensity is zero, resulting in a linear distri- 440 bution of temperature along the thermoelement due to 441 the Fourier effect (denoted by $\mathrm{F}$ in the table). In addi- 442 tion, a voltage distribution is generated due to the See- 443 beck effect (denoted by S). For case II, an electric in- 444 tensity is prescribed and the Joule effect $(\mathrm{J})$ appears. 445 Then, the temperature distribution is quadratic due to 446

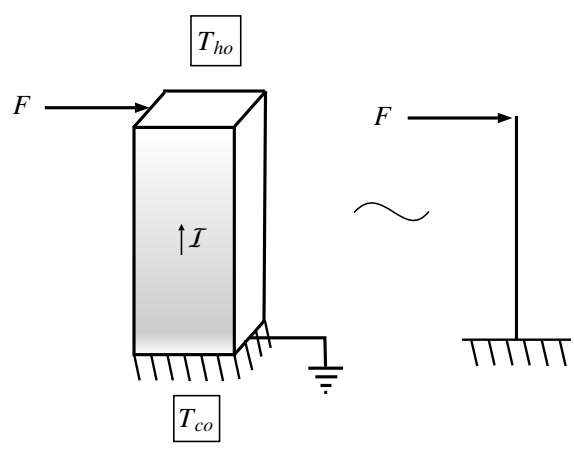

Figure 4: Geometric model and boundary conditions for validations. The 3D model (left) is reduced to a 1D beam model (right).

the heat source. For case III the temperature dependency of the $\alpha\left(T_{c}\right)$ is considered and the Thomson effect (Th) emerges. Finally, for case IV a force is applied at the end of the beam; this force represents the thermal expansion of the copper bar, see Figure 8 (top), and is proportional to: $E \alpha_{T} \Delta T$. Consequently, a bending (Bn) is observed. Due to J and Th effects, cases II, III and IV are non-linear.

\begin{tabular}{|c|l|l|}
\hline Case & Simplifications & Effects \\
\hline \hline I & $\mathcal{I}=0 ; \alpha, \gamma, \kappa=c t$ & F, S \\
\hline II & $\mathcal{I}=2[A] ; \alpha, \gamma, \kappa=c t$ & F, S, J \\
\hline III & $\mathcal{I}=2[A] ; \alpha\left(T_{c}\right) ; \gamma, \kappa=c t$ & F, S, J, Th \\
\hline IV & $\mathcal{I}=2[A] ; \alpha, \gamma, \kappa=c t ; F=1[\mathrm{~N}]$ & F, S, J, Bn \\
\hline
\end{tabular}

Table 2: One-dimensional validation cases, simplifications and considered effects: F - Fourier, S - Seebeck, J - Joule, Th - Thomson and $\mathrm{Bn}$ - bending. Constant $(c t)$ properties are obtained by using (3) with $\mathrm{T}=\left(T_{c o}+T_{h o}\right) / 2$.

Figure 5 compares analytical (lines) and numerical solutions (solid circles) for cases I to III and for three time instants $t=1,5,40$ [s]. Temperature distributions (left), voltage distributions (middle) and axial displacements (right) along the thermoelement (denoted as Distance in the figure) are represented. Each case corresponds to a row.

For case I (first row in Figure 5), the temperature distribution (left) parabolically goes from $0\left[{ }^{\circ} \mathrm{C}\right]$ at $t=0$ [s] to the linear distribution at $t=40$ [s]. Notice that at cold and hot sides the temperature is fixed and the steady state is achieved at approximately $t=40[\mathrm{~s}]$. Due to the parabolic nature of the energy balance, second in (1), the velocity of the temperature wave is infinite. For this reason, smooth curves without front wave are observed. At the steady state, a linear distribution is reached according to the Fourier law. Due to the Seebeck effect, voltage distributions (middle) are generated. 

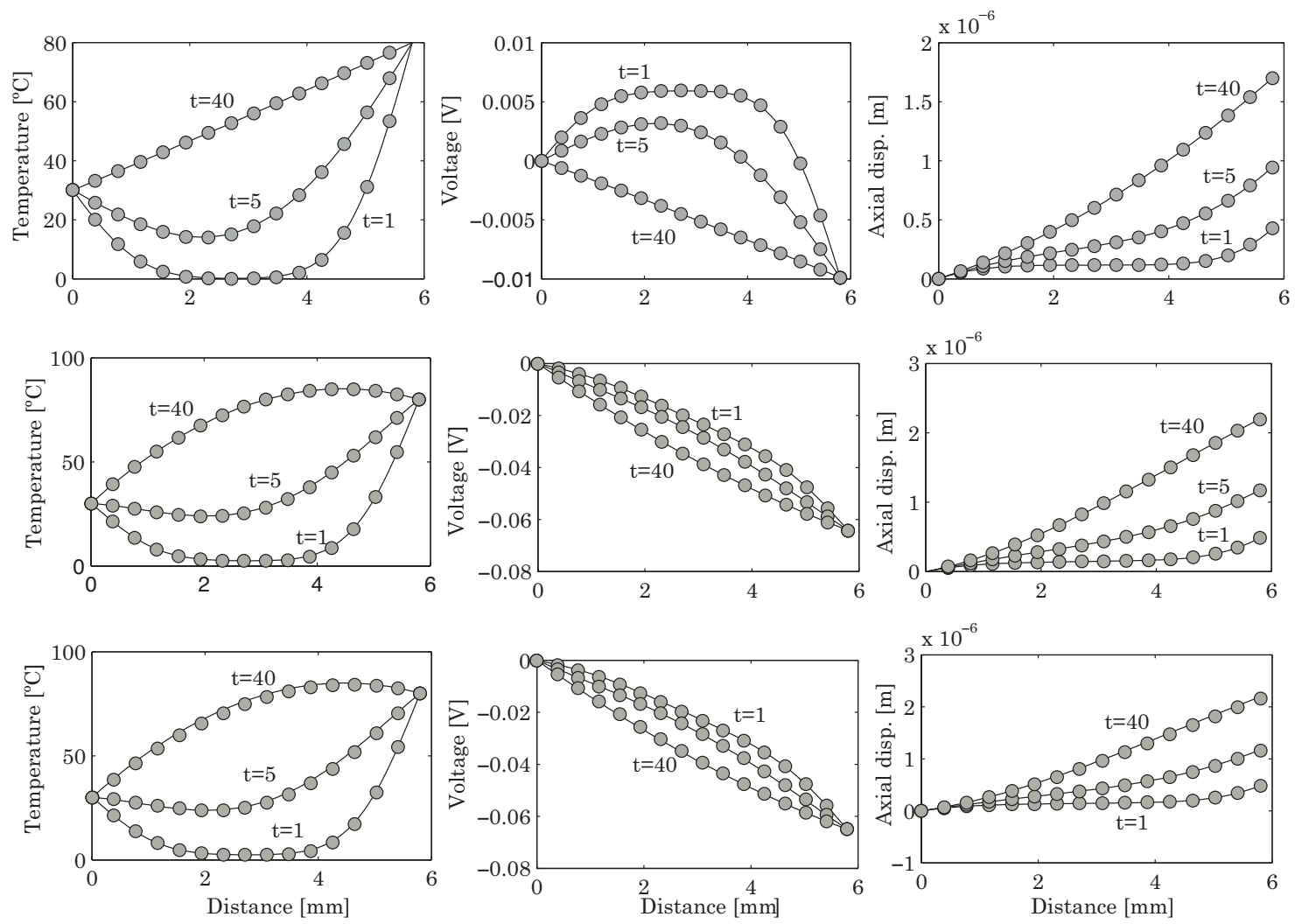

Figure 5: Analytical (lines) and numerical solutions (solid circles) for cases I to III (each case represented in a row) and for three time instants $t=1,5,40$ [s]. Temperature (left), voltage (middle) and axial displacements (right) vs. distance of thermoelement.

These distributions are proportional to the temperature 469 curves, in which the Seebeck coefficient given in Table 1470 is the constant of proportionality. Finally, the axial dis- 471 placement is represented in the right figure for the three ${ }_{472}$ instants. At the steady state, a quadratic displacement ${ }_{473}$ is obtained since it depends on the spatial integration 474 of the linear temperature distribution. In addition, the 475 displacement is proportional to the thermal expansion 476 coefficient given in Table 1. The resultant axial force is 477 zero since the mechanical boundary conditions allow a 478 free expansion of the thermoelement.

For case II (second row in Figure 5), again parabolic distributions are obtained. However, the temperature and, consequently, voltage distributions are quadratic at $t=40[\mathrm{~s}]$ since the Joule effect is present. This effect ${ }^{483}$ can be understood as a volumetric heat source that in- ${ }^{484}$ creases the temperature inside the thermoelement. For ${ }^{485}$ instance, the maximum temperature is $85\left[{ }^{\circ} \mathrm{C}\right]\left(5 \mathrm{de}-{ }^{486}\right.$ grees greater than the temperature at the hot side). Fi- ${ }^{487}$ nally, for the axial displacement a cubic distribution is ${ }^{488}$ reached at the steady state and, again, the resultant axial 489 force is zero.
The distributions for case III (third row in Figure 5) are similar to those obtained in case II since the Thomson effect involves a correction of about $1-2 \%$ of the results, as was reported in [27]. For instance, the maximum temperature inside the thermoelement is 84.24 $\left[{ }^{\circ} \mathrm{C}\right]$. The Thomson effect decreases the maximum temperature since it is an extra heat flux with opposite sign to the Joule heating. This reduction of the temperature also slightly decreases the generated voltage and axial displacement. For the temperature, there exists a small difference between numerical and analytical results due to the fact that for the analytical solution is assumed a constant Thomson coefficient in order to solve the partial differential equation.

Figure 6 shows the axial $u$ and vertical $v$ displacements and rotation $\theta$ for case IV, in which bending is present due to the application of a force $F=1[\mathrm{~N}]$, see Figure 4; only the steady state solutions are represented. Both for displacements (left axis in Figure 6) and for rotation (right axis), the agreement between closed and numerical solutions is very good.

In conclusion, Table 3 shows the maximum relative 


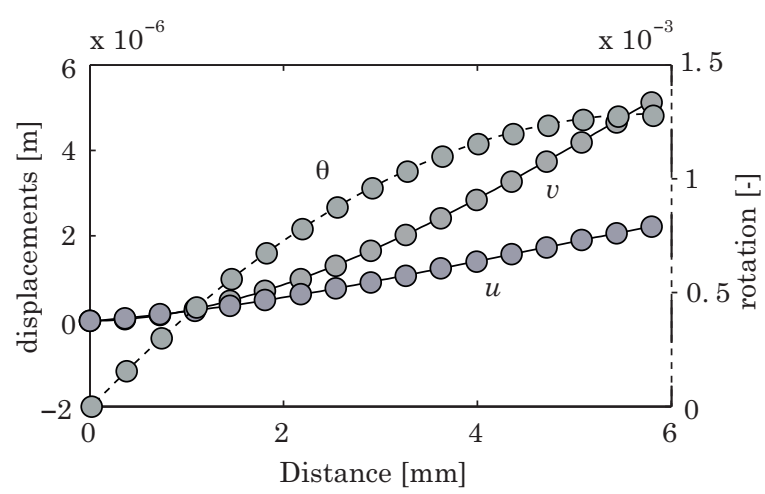

Figure 6: Analytical (lines and dashed line) and numerical solutions 510 (solid circles) for case IV. Vertical $v$ and axial $u$ displacements (left 511 axis) and rotation $\theta$ (right axis) vs. distance of thermoelement.

errors at $t=40$ [s] (steady state). For cases I, II and ${ }^{514}$ IV, the relative errors are lower than $0.1 \%$; for case III ${ }^{515}$ the errors become greater than $2 \%$. As commented, this 516 fact could be due to the calculation of the Thomson $\mathrm{co}^{-517}$ efficient: from an analytical point of view it is assumed ${ }^{518}$ to be constant and, numerically, is obtained from (3). $\quad{ }_{519}^{519}$

\begin{tabular}{|c|l|l|l|l|}
\hline Magnitude & Case I & Case II & Case III & Case IV ${ }^{52}$ \\
\hline \hline Temperature & 0.037 & 0.033 & 2.450 & 0.033 \\
\hline Voltage & 0.087 & 0.021 & 3.010 & 0.021 \\
\hline Axial disp. & 0.027 & 0.019 & 2.241 & 0.019 \\
\hline Vertical disp. & - & - & - & 0.061 \\
\hline Rotation & - & - & - & 0.062 \\
\hline
\end{tabular}

Table 3: Maximum relative errors in [\%] between analytical and nu- ${ }^{528}$ merical results at $t=40[\mathrm{~s}]$ (steady-state solution), see Figure 5.

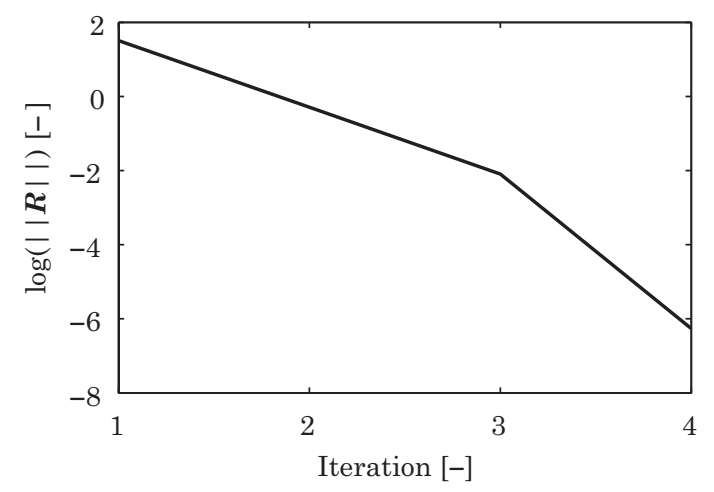

Figure 7: Study of the Newton-Raphson convergence: logarithmic residual norm vs. iteration.

As commented, cases II and III are non-linear due ${ }_{547}$ to the presence of the Joule effect. Figure 7 shows the ${ }_{548}$ logarithm of the residual norm versus the number of it- 549 erations $i$ of the Newton-Raphson algorithm. Considering $\left\|\mathcal{R}_{i+1}\right\| \leq p_{1}\left\|\mathcal{R}_{i}\right\|^{p_{2}}$, where $p_{1}$ and $p_{2}$ are positive constants, it is observed that $p_{2}>1$ and a super-linear convergence is achieved.

\section{Comparisons with 3D FE model}

The main purpose of this section is to compare the present beam formulation with the $3 \mathrm{D}$ one reported in [5]. Then, CPU times and relative errors are calculated and the advantages and drawbacks of the proposed beam formulation are highlighted.

In order to perform this comparison, a pulsed Peltier device as that described in [16] is simulated. This device is composed of 127 thermocouples electrically connected in series and thermally in parallel. A thermocouple is a pair of two p- and n-type thermoelements connected by copper $C u$ bars and with tin $S n$-Pb solders, as shown in Figure 8 (top). The material properties of the thermoelements are given in Table 1; the ones of $\mathrm{Cu}, \mathrm{Sn}-\mathrm{Pb}$ and the dimensions of the thermocouple are reported in [16].

For the 3D model, the full thermocouple is modeled using the structured mesh shown in Figure 8 (top). For the beam model, only the line of centroids of each material is modeled. This geometry is highlighted in the figure by superposing solid black lines in the 3D mesh. Obviously, the number of finite elements required to model the thermocouple is highly reduced using the beam formulation. Boundary conditions are also represented in the figure: mechanically, the devices is fixed at the hot side (bottom in the figure); thermally, the temperatures at both sides are $T_{c o}=20, T_{h o}=50\left[{ }^{\circ} \mathrm{C}\right]$; electrically, the voltage is set to zero at the middle of the horizontal $\mathrm{Cu}$ beams and an electric intensity $I=1$ $[\mathrm{A}]$ is prescribed.

Figure 9 shows the h-convergence of the Coefficient Of Performance (COP), top figure, and maximum Von Mises (VM) stress inside the thermocouple (middle) versus the number of FE nodes, for both 3D (solid line) and beam (dashed line) steady-state solutions. Notice that the calculation of COP and VM involves thermoelectric and thermoelastic variables, respectively: all the couplings are required. Finally, the bottom figure represents the CPU time for each calculation.

For the 3D model, the COP converges with approximately 4500 nodes whereas the VM requires 6000 nodes. This difference in the number of nodes is due to the vectorial nature of the thermoelectric variables and the second rank of the stress tensor. For a proper calculation of both variables a CPU time of 50 [s] is employed. 


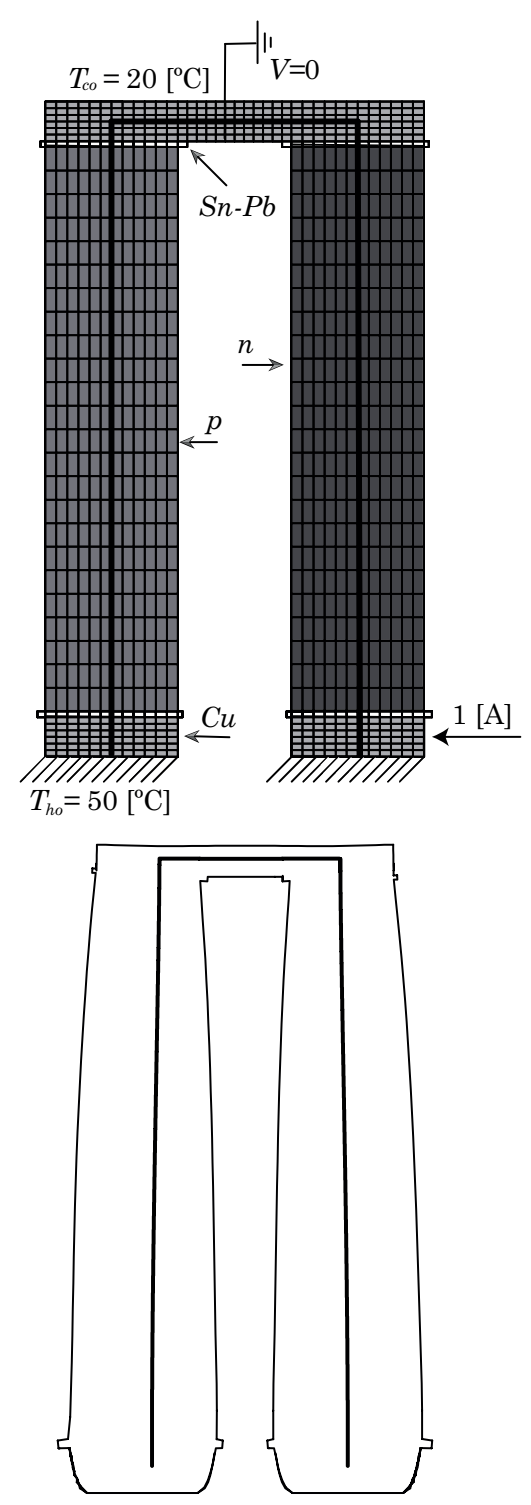

Figure 8: Top: 3D and beam (highlighted in solid black lines) meshes of a thermocouple, composed of four materials: $\mathrm{n}$ - and $\mathrm{p}$-type thermoelements, copper $\mathrm{Cu}$ bars and tin $\mathrm{Sn}-\mathrm{Pb}$ solders. Mechanical, thermal and electrical boundary conditions also represented. Bottom: Outline of the deformed 3D and beam meshes $($ zoom $\times 500)$.

For the beam model, the requirement of nodes (approximately 500) and consequently the CPU time (lower than $3[\mathrm{~s}]$ ) decreases since a $1 \mathrm{D}$ mesh is used. The main advantage of the beam formulation is the reduction of CPU time; in contrast, the beam model has a lower accuracy. The relative errors between 3D and beam formulations are $e_{C O P}=6.6 \%$ and $e_{V M}=12.6 \%$.

To sum up, the lower CPU time for the beam model 568 could permit the application of the present formulation

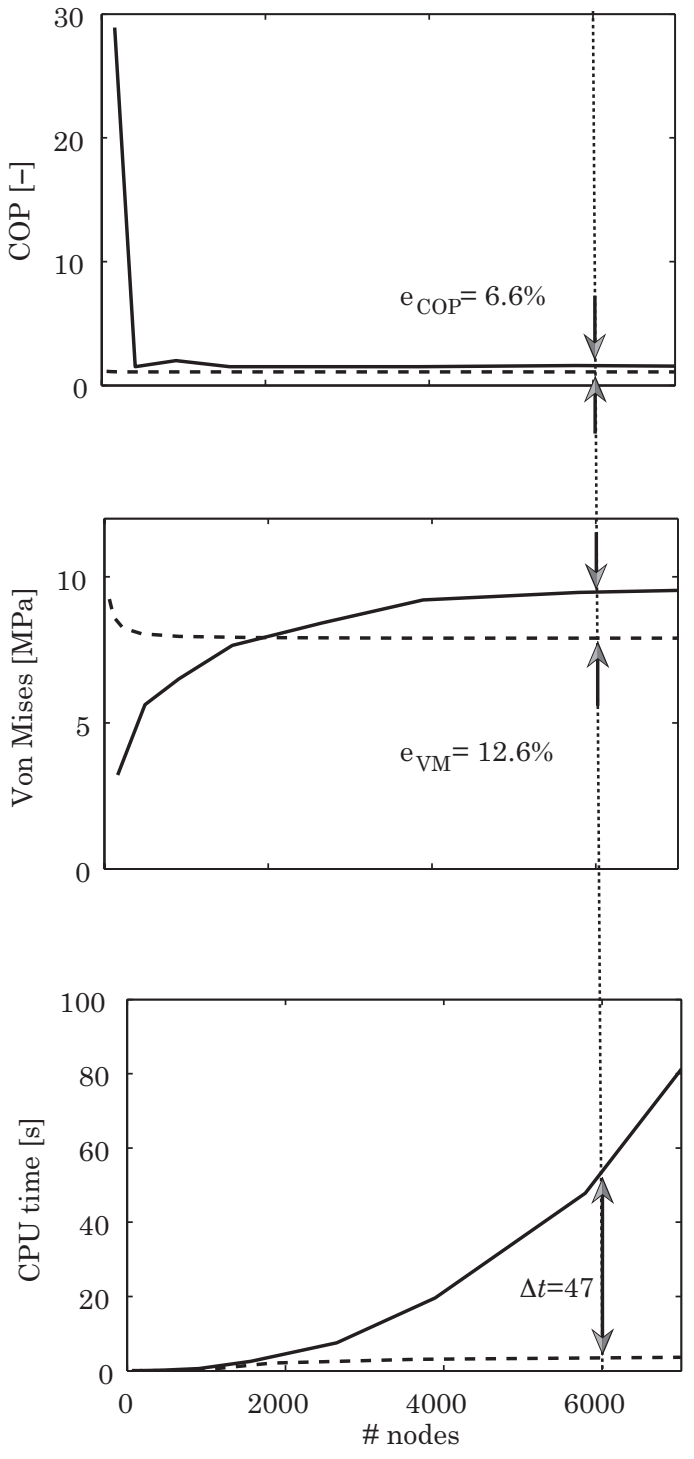

Figure 9: h-convergence and CPU time for both 3D (solid line) and beam (dashed line) steady-state solutions. COP (top), Von Mises stress (middle) and CPU time (bottom) vs. number of nodes. Relative errors between 3D and beam models denoted by $e$.

in sensitivity analyses and optimizations, which require many evaluations to obtain the final results.

Figure 10 shows the distributions of voltage (topleft), temperature (top-right), horizontal (bottom-left) and vertical (bottom-right) displacements versus the distance. Now, solid lines represent the 3D calculations and, again, solid circles the beam solutions.

Regarding voltage, the agreement between 3D and beam results is very good except in the bottom $\mathrm{Cu}$ bar. Obviously, the beam model shows an idealization for which all the horizontal electric flux along the bar flows 
vertically through the thermoelement. On the contrary, 620 the 3D model captures the rotational effects that are present at both bottom corner of the thermoelement. This limitation of the beam model is the cause of the ${ }_{622}$ larger relative error in the COP calculation, as com- ${ }_{62}$ mented in the previous paragraphs.

In terms of temperature, a good agreement between both solutions is achieved. Minor differences are ob- ${ }_{624}$ served due to the higher potential drop (consequently, higher Joule heating) predicted by the 3D model.

On the one hand, the horizontal displacement 626 (bottom-left) is due to the thermal contraction of the ${ }^{627}$ copper bar at the top of the devices that results in a ${ }_{629}^{628}$ bending of both thermoelements, see Figure 8 (bottom) ${ }_{630}$ where an outline of the deformed 3D and beam meshes ${ }^{631}$ are represented. On the other hand, the vertical dis- ${ }^{632}$ placement is due to the thermal expansion of the device. ${ }_{634}{ }^{633}$ Notice that the reference temperature is assumed to be 635 $25\left[{ }^{\circ} \mathrm{C}\right]$ and, therefore, both thermal expansion and con- ${ }^{636}$ traction are present. The comparison between both, 3D ${ }^{637}$ and beam solutions, shows a reasonable agreement tak- ${ }^{638}$ ing into account the limitations of the 1D formulation. $\quad 640$

Nevertheless, the remarkable changes in the cross ${ }^{641}$ sections observed for the 3D model in Figure 8 (bot- ${ }_{643}^{642}$ tom) can not be captured by the beam model since, ac- ${ }_{644}$ cording to $\boldsymbol{A l}$, the cross sections in the beam model are 645 assumed as rigid bodies and, consequently, they can not ${ }^{646}$ be deformed.

\section{Conclusions}

This work presents a dynamic, non-linear and fully ${ }^{652}$ coupled finite element formulation based on the Tim- 654 oshenko beam theory to study elasto-thermoelectricity. ${ }^{655}$ The formulation is implemented in the research code ${ }^{656}$ FEAP and is validated using $1 \mathrm{D}$ analytical solutions. ${ }_{658}^{657}$ Then, this formulation is used to model a pulsed Peltier 659 device and the results are compared with 3D FE solu- ${ }^{660}$ tions.

The main advantage of the beam model is the low ${ }_{663}^{662}$ computational cost. For instance, a reduction of 47 [s] 664 with respect to a 3D model can be achieved for the mod- ${ }^{665}$ eling of a pulsed Peltier devices. In contrast, the main ${ }^{666}$ drawback is the inherent error due to the 1D limitations ${ }_{668}$ of the beam theory. In this regard, a maximum relative ${ }_{669}$ COP error of $6.6 \%$ between both models is found. $\quad 670$

In conclusion, the combination of the beam element ${ }_{672}^{671}$ to obtain coarse results and the $3 \mathrm{D}$ model to calculate 673 details of the Peltier devices is a methodology that could 674 provide good results with a comprehensive CPU time. ${ }^{675}$ This combination could be used in future optimizations ${ }_{677}^{676}$ and sensitivity analyses that require many evaluations. $\quad 678$

\section{Acknowledgments}

The authors of the present work would like to acknowledge to the students Jorge Segarra Tamarit and Alfredo Martí García for their collaboration.

\section{References}

[1] R.L. Taylor. FEAP A Finite Element Analysis Program: User Manual. University of California, Berkeley, 2010. http://www.ce.berkeley.edu/feap.

[2] J.L. Pérez-Aparicio, R. Palma, and R.L. Taylor. Multiphysics and thermodynamic formulations for equilibrium and nonequilibrium interactions: non-linear finite elements applied to multi-coupled active materials. Arch Comput Methods Eng, accepted:-, 2015.

[3] D. Zhao and G. Tan. A review of thermoelectric cooling: Materials, modeling and applications. Applied Thermal Engineering, 66:15-24, 2014.

[4] J.L. Pérez-Aparicio, R.L. Taylor, and D. Gavela. Finite element analysis of nonlinear fully coupled thermoelectric materials. Computational Mechanics, 40:35-45, 2007.

[5] J.L. Pérez-Aparicio, R. Palma, and R.L. Taylor. Finite element analysis and material sensitivity of Peltier thermoelectric cells coolers. International Journal of Heat and Mass Transfer, 55:1363-1374, 2012.

[6] R. Palma, J.L. Pérez-Aparicio, and R.L. Taylor. Non-linear finite element formulation applied to thermoelectric materials under hyperbolic heat conduction model. Computer Method in Applied Mechanics and Engineering, 213-216:93-103, 2012.

[7] R. Palma, J.L. Pérez-Aparicio, and R. Bravo. Study of hysteretic thermoelectric behavior in photovoltaic materials using the finite element method, extended thermodynamics and inverse problems. Energy Conversion and Managament, 65:557-563, 2013.

[8] I.J. Ferrer, P. Díaz-Chao, A. Pascual, and C. Sánchez. Hysteresis-like behaviour of the thermoelectric voltage in photovoltaic materials. Thin Solid Films, 511-512:177-181, 2006.

[9] E.E. Antonova and D.C. Looman. Finite elements for thermoelectric device analysis in ansys. In International Conference on Thermoelectrics, 2005.

[10] Y. Shi, Z. Zhu, Y.n Deng, W. Zhu, X. Chen, and Y. Zhao. A real-sized three-dimensional numerical model of thermoelectric generators at a given thermal input and matched load resistance. Energy Conversion and Management, 101:713-720, 2015.

[11] G. Wu and X. Yu. A holistic 3D finite element simulation model for thermoelectric power generator element. Energy Conversion and Management, 86:99-110, 2014.

[12] M. Jaegle. Multiphysics simulation of thermoelectric systems modeling of peltier - cooling and thermoelectric generation. In Proceedings of the COMSOL, 2008.

[13] S. Turenne, T. Clin, D. Vasilevskiy, and R.A. Masut. Finite element thermomechanical modeling of large area thermoelectric generators based on bismuth telluride alloys. J Electron Mater, 39:1926-1933, 2010.

[14] M. Picard, S. Turenne, D. Vasilevskiy, and R.A. Masut. Numerical simulation of performance and thermomechanical behavior of thermoelectric modules with segmented bismuth-telluridebased legs. J Electron Mater, 42:2343-2349, 2013.

[15] Y. Wu, T. Ming, X. Li, T. Pan, K. Peng, and X. Luo. Numerical simulations on the temperature gradient and thermal stress of a thermoelectric power generator. Energy Conversion and Management, 88:915-927, 2014. 

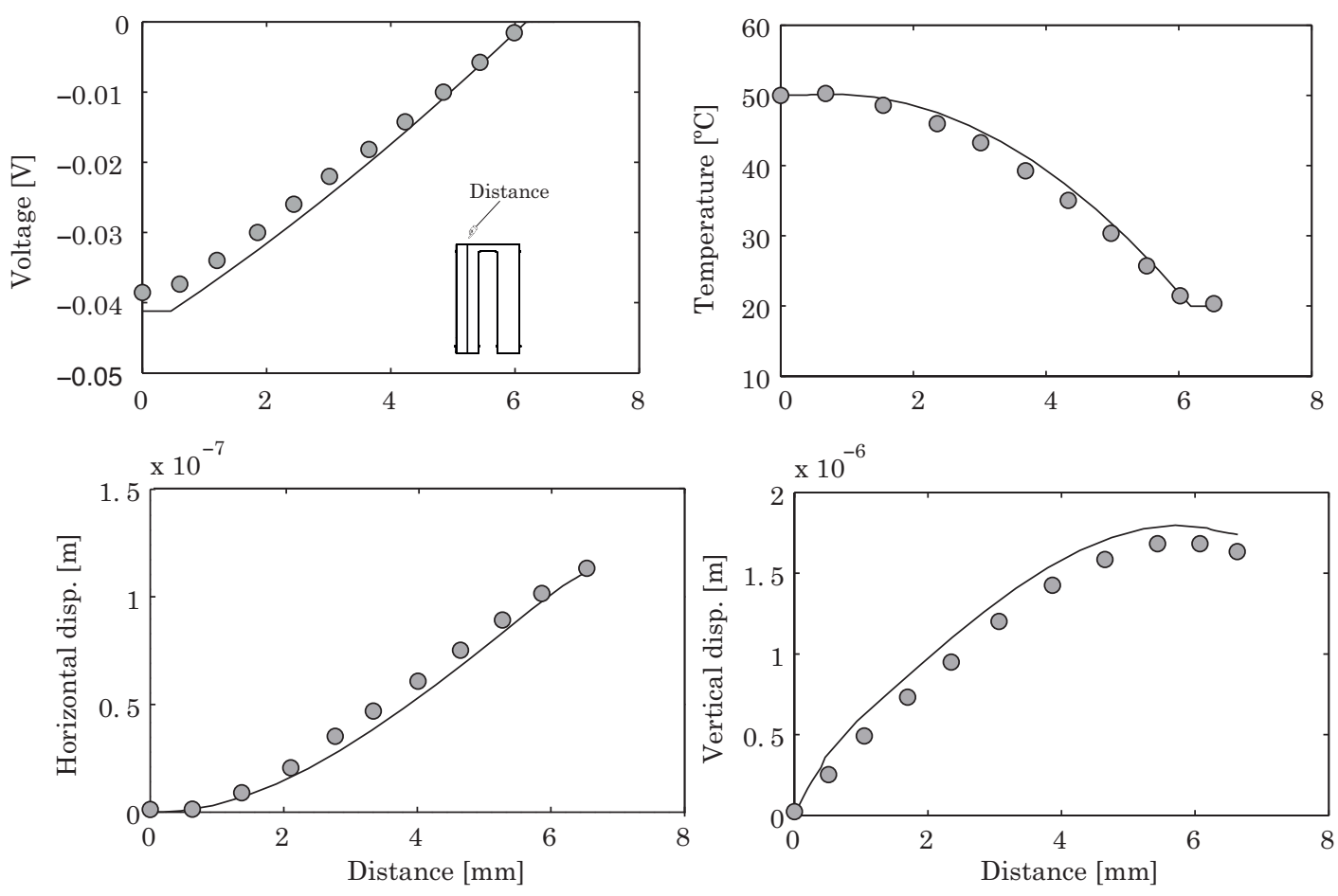

Figure 10: Distributions of voltage (top-left), temperature (top-right), horizontal (bottom-left) and vertical (bottom-right) displacements vs. distance. 3D calculations in solid lines and beam in solid circles.

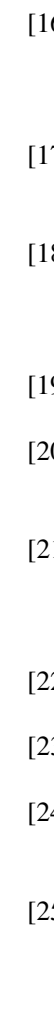

[16] J.L. Pérez-Aparicio, R. Palma, and P. Moreno-Navarro. Elasto- 710 thermoelectric non-linear, fully coupled, and dynamic finite el- 711 ement analysis of pulsed thermoelectrics. Applied Thermal En- 712 gineering, 107:398-409, 2016.

17] R. Poya, A.J. Gil, and P.D. Ledger. A computational frame- 714 work for the analysis of linear piezoelectric beams using hp- 715 FEM. Computers $\mathcal{E}$ Structures, 152:155-172, 2015.

18] V. Kutis, J. Murín, R. Belák, and J. Paulech. Beam element with 717 spatial variation of material properties for multiphysics analysis of functionally graded materials. Computers $\mathcal{E}$ Structures, 89:1192-1205, 2011.

19] M.A. Biot. Thermoelasticity and irreversible thermodynamics. Journal of Applied Physics, 27(3):240-253, 1956.

[20] X. Guo, Y.-B. Yi, and S. Pourkamali. A finite element analysis of thermoelastic damping in vented MEMS beam resonators. International Journal of Mechanical Sciences, 74:73-82, 2013.

$21]$ D.V. Parayil, S.S. Kularni, and D.N. Pawaskar. Analytical and numerical solutions for thick beams with thermoelastic damping. International Journal of Mechanical Sciences, 94-95:10$19,2015$.

22] S. Timoshenko. Strength of Materials: Elementary Theory and Problems. D. Van Nostrand Company, Inc., 1951.

23] K.D. Hjelmstad, editor. Fundamentals of Structural Mechanics. Springer Science + Business Media, Inc., 2005.

24] R.A. Sauer and J.C. Mergel. A geometrically exact finite beam element formulation for thin film adhesion and debonding. $\mathrm{Fi}$ nite Elements in Analysis and Design, 86:120-135, 2014.

25] S. Gao, B. Liang, and E. Vidal-Salle. Development of a new 3D beam element with section changes: The first step for large scale textile modelling. Finite Elements in Analysis and Design, 104:80-88, 2015
[26] R.E. Erkmen and M.M. Attard. Displacement-based finite element formulations for material-nonlinear analysis of composite beams and treatment of locking behaviour. Finite Elements in Analysis and Design, 47:1293-1305, 2011.

[27] M.J. Huang, R.H. Yen, and A.B. Wang. The influence of the Thomson effect on the performance of a thermoelectric cooler. International Journal of Heat and Mass Transfer, 48:413-318, 2005. 